\title{
CRIB II score versus gestational age and birth weight in preterm infant mortality prediction: who will win the bet?
}

BY MLADEN JAŠIĆ, NADA SINDIČIĆ DESSARDO, SANDRO DESSARDO, KORALJKA MANESTAR RUKAVINA

\section{Abstract}

Introduction. In neonatology, various illness severity scores have been developed to predict mortality and morbidity risk in neonates. The aim of our study was to validate the ability of the 'Clinical Risk Index for Babies' (CRIB) II score to predict mortality in neonates born before 32 weeks' gestation in a level 3 neonatal intensive care unit (NICU), setting.

Materials and Methods. Prospective birth cohort study including all liveborn neonates of 32 weeks' gestation or less. . CRIB II score was calculated and the predicted mortality was compared with the observed mortality. Discrimination (the ability of the score to correctly predict survival or death) was assessed by calculating the receiver operating characteristic curve (ROC curve) and its associated area under the curve (AUC). 
Results. The ROC curve analysis in our study showed that the AUC was 0.9008 suggesting that mortality prediction was $90 \%$ accurate for all infants. Sensitivity and specificity were $77 \%$ and $88 \%$ respectively. In our study population, the CRIB II score appears to be more accurate than gestational age and birth weight in predicting mortality.

Conclusions. The CRIB II scoring system is a useful tool for predicting mortality and morbidity in NICUs, and also a useful tool for evaluating the variations in mortality and other outcomes seen between different NICUs.

Key words: CRIB, CRIB II, mortality, neonates, outcome, prematurity, scoring system

\section{Introduction}

In neonatology, various illness severity scores have been developed during the last two decades with the purpose of predicting mortality and morbidity in neonates. Moreover, the use of scoring systems is mandatory in order to effectively compare performances between neonatal intensive care units (NICUs), in regard to variations in mortality and morbidity between different NICUs. $(1,2)$

The Clinical Risk Index for Babies (CRIB) score is a risk-adjustment instrument widely used in NICUs. (3)

It was developed more than twenty years ago and is based upon six variables. Recently, its appropriateness has been questioned since mortality in NICUs has fallen because of improvement in therapy and monitoring. (4)

Furthermore, CRIB includes two potentially questionable parameters: 1 ) the fraction of inspired oxygen $\left(\mathrm{FiO}_{2}\right)$, which is not a true physiological measure because it is determined by the care team; and 2) data collectected up to 12 hours after admission, thus potentially introducing early treatment bias. 
In order to overcome these issues, a new, simplified and recalibrated five-item CRIB II score was developed in 2003. (5)

The aim of our study was to validate the ability of CRIB II score to predict mortality in neonates born before 32 weeks' gestation in a level 3 NICU setting. This study is the first prospective analysis of CRIB II score in the Croatian neonatal population.

\section{Materials and Methods}

This prospective birth cohort study was performed at the tertiary Neonatal Intensive Care Unit, Department of Gynaecology and Obstetrics, Division of Neonatology, University Hospital Rijeka, during a five-year-period (January $1^{\text {st }} 2008$ - December $31^{\text {st }} 2012$ ).

The study population included all live-born neonates of 32 weeks' gestation or less. Exclusion criteria were: 1) less than 23 weeks' gestation; 2) admission to NICU more than 12 hours after delivery; 3) presence of a lethal congenital malformation; 4) death within the first 12 hours of life.Gestational age was calculated based on the first day of the last menstrual period (LMP). In cases where LMP was not known, gestational age was assessed using obstetric ultrasonography. The birth weight was recorded in NICU, immediately after admission, using an electronic scale with a sensitivity of 10 grams. Capillary blood gas analysis was performed in all infants. The body temperature was measured using a digital thermometer with a sensitivity of $0.1^{\circ} \mathrm{C}$. The above mentioned parameters were used to calculate the CRIB II score.

The measured outcome was in-hospital mortality. Predicted mortality was compared with observed mortality. A logistic model was used to analyse the prediction of mortality using the CRIB II score on admission. Discrimination (the ability of the score to correctly predict survival or death) was assessed by calculating the receiver operating characteristic curve (ROC curve) and its associated area under the curve (AUC). An AUC value of 0.5 or less indicates no ability to discriminate, whereas larger values indicate increasing ability. A value of 0.8 is considered 
good. $(6,7)$

\section{Results}

One hundred and fifty three infants, which fulfilled the criteria for the study, were admitted to NICU during a 5-year period (males: 84 (55\%), females: $69(45 \%))$.

The average gestational age was $27.68 \pm 2.32$ weeks (median 28.0o, range 23.00-31.00) and average birth weight was 1063.11 $\pm 369.24 \mathrm{~g}$ (median 1040.00, range 390.00-1900.00). The CRIB II score was 9.03 \pm 4.28 (median 9.00, range 1.00-19.00). One hundred and nineteen neonates survived (78\%) and 34 died (22\%).

ROC curve analysis of mortality prediction capability of CRIB II showed that the AUC was 0.9008 (SE 0.0264, 95\% CI: 0.84204-0.94317) suggesting that mortality prediction was $90 \%$ accurate for all infants (figure 1). Sensitivity was $77 \%$ and specificity $88 \%$, respectively.

ROC curve analysis of mortality prediction capability based on birth weight showed that the AUC was 0.842 (SE 0.0364, 95\% CI: 0.7740.896), while the ROC curve analysis for gestational age showed that the AUC was 0.840 (SE 0.0395, 95\% CI 0.772-0.894) (figure 1)

Med Calc 9.3.9.0. software was used for data analysis (MedCalc, Mariakerke, Belgium).

\section{Discussion}

The main finding emerging from our results is that CRIB II is a reliable tool for mortality prediction in a preterm population, within a level 3 NICU setting, and performs better than gestational age or birth weight.

Nevertheless, there are some possible study limitations. The most important is related to the small number of enrolled infants, particularly if we consider the number of extremely low birth weight (ELBW) infants, 
which was only $15 \%$, too few to examine this sub-group separately.

According to Fleisher and co-workers, the properties of a scoring system should be: " (1) ease of use; (2) applicability early in the course of hospitalisation; (3) ability to reproducibly predict mortality, specific morbidities, or cost for various categories of neonates; (4) usefulness for all groups of neonates to be described". (8)

The ability of a score to differentiate between infants with different outcomes (discrimination) is also important, as good calibration cannot be achieved without good discrimination. (1) Discrimination is measured by the area under the receiver operating characteristic curve obtained by plotting the true positive rate against the false positive rate for the full range of values. $(7,9)$

The area under this curve indicates the overall discriminatory ability of a scoring system. An ideal test would have an area of 1.0 (no false positive or false negative results), whereas a score no better than chance alone has the value 0.5 . A value above 0.8 is often considered as an indicator that the score may be useful in practice. $(6,7)$

The CRIB score was created to predict mortality for infants born before 32 weeks' gestation and was derived using data from infants admitted to four UK tertiary neonatal units in the period 1988 - 1990. (3) CRIB II, an amended and potentially improved version of CRIB, was published in 2003. (5) The new score was intended to improve predictions for smaller, very premature infants and to exclude variables that could be influenced by care given to the infant. $(1,5)$

So far, published analyses of the mortality prediction capability of CRIB II have showed controversial results, very much dependent on the study setting. Reports are swinging between the conclusion that CRIB-II does not result in improved estimation of mortality risk in very low birth weight (VLBW) infants as compared to CRIB, birth weight or gestational age, to totally opposite statements that CRIB and CRIB-II show similar accuracy values in predicting in-hospital neonatal mortality in VLBW infants. Nevertheless, it appears that neither score offers a particular 
advantage in predicting mortality, as compared to gestational age or birth weight. $(10,11)$

The ROC curve analysis in our study showed that the AUC was 0.9008 suggesting that mortality prediction was $90 \%$ accurate for all infants. Sensitivity and specificity were $77 \%$ and $88 \%$ respectively. In the study of Mohkam et al. (4) conducted in Tehran, Iran, CRIB II AUC was 0.698 with a sensitivity of $69.6 \%$ and specificity of $63.0 \%$. The mortality prediction using CRIB II score was better in our infants. Results similar to ours were published by Rastogi et al. (6) in 2010; they showed that CRIB II correctly predicted adverse outcomes in 90.3\% of Indian preterm infants, AUC 0.9032 (SE 0.0345, 95\% CI: 0.83553-0.97096).

Obviously, the prediction capability of CRIB II, as with other scoring systems, depends on the characteristics of the NICU setting. Our study results showed a considerably better prediction capability of CRIB II when compared to gestational age or birth weight.

In a recent study Gooden and co-workers found that in resource-poor neonatal settings, where mortality of VLBW infants is high, there may be no benefit of additional variables and scores other than birth weight, in order to increase the capability of mortality prediction. (12)

Greenwood, in a study evaluating CRIB-II as a predictor of moderate to severe functional disability (FD) in very premature infants, concluded that, although CRIB-II improved prediction of mortality, it did not perform better than gestational age or BW in predicting FD. (13)

Even if not a perfect neonatal scoring system, CRIB II could have a role as a reliable illness severity index in different research settings in the NICU environment. $(14,15)$

Manktelow and co-workers, aiming to validate the predictive value of different versions of CRIB II, came to a conclusion that CRIB II could be used in benchmarking mortality in neonatal intensive care units, even when some variables are omitted. (16) 


\section{Conclusion}

The CRIB II scoring system is a useful tool for predicting mortality and morbidity in NICUs, and also a useful tool for evaluating variations in mortality and other outcomes seen between different NICUs. The ability of CRIB II to predict mortality better than birth weight or gestational age depends on the specific neonatal setting and is clearly influenced by the overall level of care and quality of neonatal resources.

\section{References}

1. Dorling JS, Field DJ, Manktelow B. Neonatal disease severity scoring systems. Arch Dis Child Fetal Neonatal Ed 2005; 90:F11-F16.

2. Signorini DF, Weir NU. Any variability in outcome comparisons adjusted for case mix must be accounted for. BMJ 1999;318(7176):128.

3. The International Neonatal Network. The CRIB (clinical risk index for babies) score: a tool for assessing initial neonatal risk and comparing performance of neonatal intensive care units. Lancet 1993;342:193-8.

4. Mohkam M, Afjeii A, Payandeh P, Zadkarami M, Kazemian M, Fakhraii $\mathrm{H}$, et al. A comparison of CRIB, CRIB II, SNAP, SNAPII and SNAP-PE scores for prediction of mortality in critically ill neonates. MJIRI 2011;24(4):193-9.

5. Parry G, Tucker J, Tarnow-Mordi W. CRIB II: an update of the clinical risk index for babies score. Lancet 2003;361:1789-91.

6. Rastogi PK, Sreenivas V, Kumar N. Validation of CRIB II for prediction of mortality in premature babies. Indian Pediatrics 2010;47:145-7.

7. Hanley JA, Mcneil BJ. The meaning and the use of the area under a receiver operating characteristic (ROC) curve. Radiology 1982;143:29-36.

8. Fleisher BE, Murthy L, Lee S, Constantinou JC, Benitz WE, Stevenson DK. Neonatal severity of illness scoring systems: a comparison. Clin Pediatr 1997;36:223-7.

9. Van Erkel AR, Pattynama PMT. Receiver Operating Characteristic (ROC) analysis: basic principles and applications in radiology. Eur J Radiol 1998;27:88-94. 
10. Bührer C, Metze B, Obladen M. CRIB, CRIB-II, birth weight or gestational age to assess mortality risk in very low birth weight infants? Acta Paediatr 2008; 97(7):899-903.

11. De Felice C, Del Vecchio A, Latini G. Evaluating illness severity for very low birth weight infants: CRIB or CRIB-II? J Matern Fetal Neonatal Med 2005;17(4):257-60.

12. Gooden M, Younger N, Trotman H. What is the Best Predictor of Mortality in a Very Low Birth Weight Infant Population with a High Mortality Rate in a Medical Setting with Limited Resources? Am J Perinatol 2014;31(6):441-6.

13. Greenwood S1, Abdel-Latif ME, Bajuk B, Lui K; NSW and ACT Neonatal Intensive Care Units Audit Group. Can the early condition at admission of a high-risk infant aid in the prediction of mortality and poor neurodevelopmental outcome? A population study in Australia. J Paediatr Child Health 2012;48(7):588-95.

14. 14. Miletin J, Pichova K, Doyle S, Dempsey EM. Serum cortisol values, superior vena cava flow and illness severity scores in very low birth weight infants. J Perinatol 2010;30(8):522-6.

15. Zhang Y, Chan GS, Tracy MB, Lee QY, Hinder M, Savkin AV, et al. Spectral analysis of systemic and cerebral cardiovascular variabilities in preterm infants: relationship with clinical risk index for babies (CRIB). Physiol Meas 2011;32(12):1913-28.

16. Manktelow BN, Draper ES, Field D. Predicting neonatal mortality among very preterm infants: a comparison of three versions of the CRIB score. Arch Dis Child Fetal Neonatal E 2010; 95(1):F9-F13. 


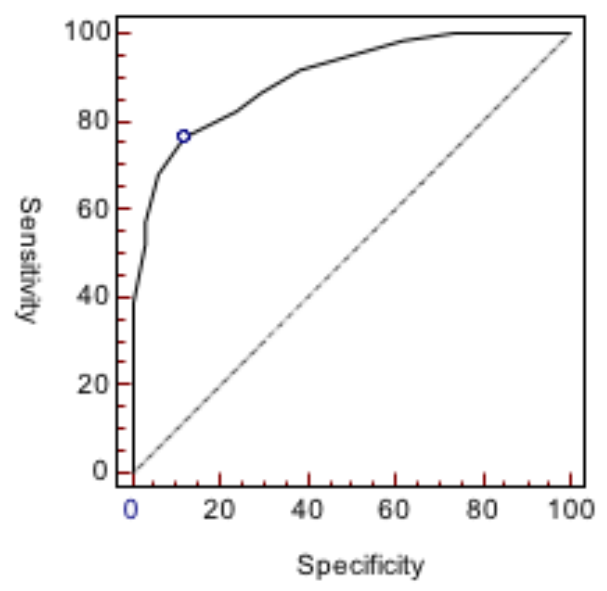

Figure 1. Mortality prediction on ROC curve with CRIB II; AUC 0.9008 (SE 0.0264, 95\% CI: 0.84204-0.94317)

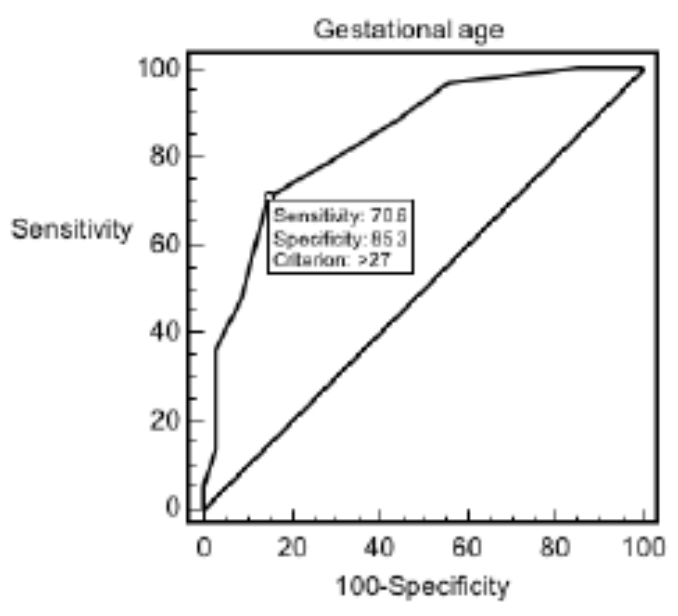

Figure 2. Mortality prediction with gestational age on ROC curve; AUC 0.840 (SE 0.0395, 95\% CI 0.772-0.894).

Mladen Jašić

Department of Paediatrics, General Hospital Pula, Pula, Croatia

Nada Sindičić Dessardo, Sandro Dessardo,

Department of Paediatrics, University of Rijeka, Rijeka, Croatia

Koraljka Manestar Rukavina

Institute of Neonatology, Department of Gynaecology and Obstetrics, University Hospital Rijeka, Rijeka, Croatia

Corresponding author

Nada Sindičić Dessardo 
Department of Paediatrics

University of Rijeka

Braće Branchetta 20, HR-5100o Rijeka, Croatia

Phone: +385916157070

E-mail: sindin2@net.hr

Article printed from Signa Vitae: http://www.signavitae.com

URL to article: http://www.signavitae.com/2016/o5/crib-iiscore-versus-gestational-age-and-birth-weight-in-preterminfant-mortality-prediction-who-will-win-the-bet/

Copyright (C) 2015 Signa Vitae. All rights reserved. 